\title{
La Salud Infantil en Chile durante la Década del Setenta
}

\author{
Drs. Ana María Kaempfer R. (1) y Encsto Medina L (2)
}

\section{Children's Health in Chile from 1970 to 1980.}

\begin{abstract}
Changes in the Chilean health situation during the period of time 1970--1980 are discussed in this report. The most important feature was the decrease of the death risk in children mainly in the $]$ month to 5 years age group. The downward trend of the death risk was observed along the country in urban and rural areas. There also was a fall in the number of cases of infectious discases with vaccine systems and in the number of diseases noticed by the mothers in home surveys.

As a consequence of these changes, tespiratory and infections discascs decreased their telative importance from $58 \%$ to $25 \%$ out of total children deaths while the perinatal problemsincressed from $17 \%$ to $30 \%$ and congenital pathology from 4 to $10 \%$.

The downward tend of morbidity and mortality in Chile was not associated with the economic situation of the country nor the changes in national organization. It can be estimated that one third of the decline is related to the changes in the type and number of children as a result of the family planning programs. Two thirds aze related to the progress in medical care with an increased number of medical visits, well baby clinic controls, hospital admissions, birth in hospital, vaccination and malnutrition control programs.
\end{abstract}

El hecho más destacado de lo ocurrido en salud en Chile en la década de 1970 fue el significativo descenso del riesgo de muerte infantil (1)(2). Esta disminución puede parecer paradojal, dado el clásico concepto de relación inversa entre mortalidad infantil $y$ nivel de vida observada en Chile y en otros países por una parte (3) (4) (5) (6) y los problemas económicos observados en el pás durante este periodo. Todavía más paradojal resulta el hecho si se considera que el descenso del rjesgo fue considerablemente mayor que el observado para América Latina como conjunto. Por las razones anteriores nos pareció de interés estudiar en detalle la situación de salud infantil y los factores condicionantes de ella en el período comprendido entre 1970 y 1980.

\section{MATERIAL Y METODO}

Para el análisis de la situación chilena se ha dispuesto de información procedente de diversas fuentes. Entre ellas destacan las del Instituto Nacional de Estadistica en materias demográficas, de salud, económicas y educacionales ( 7 a 12); las del Ministerio de Salud de tipo biodemográfico (13) (14) (15), de morbilidad y de recursos (16) (17)

(1) Jefe, División de Medicina Preventiva y Salud Matemoinfantil, Escuela de Salud Pública, Facultad de Medi cina, Universidad de Chile.

(2) Dircetor, Escucla de Salud Pública, Facultad de Medicina, Universidad de Chile.
(18) (19); las provenientes del Servicio de Seguro Social (20); las de CONPAN en diversos análisis relativos a nutrición (21); del Colegio Médico respecto a características de la profesión(22); del Ministerio de Hacienda en materias vinculadas a la situación económica general o a los recursos financieros del sector salud (23) (24) y estudios diversos realizados en la Escuela de Salud Pública de la Universidad de Chile (25 a 49) y en otras partes (50 a 56$)$.

Se recolectaron los datos disponibles para el decenio 1970-1980, como igualmente información de 1960 y 1965 para propósitos comparativos de las tendencias observadas. Los datos se organizaron en forma que permitieran el análisis de lo ocurrido con la patología y las variaciones observadas en la atención de salud y en factores condicionantes de tipo demográfico, económico-social, ambiental, educacional y nutricional.

\section{RESULTADOS}

\section{1) Magnitud de la población infantil}

Ln primer hecho destacado de lo ocurrido durante el período $1970-1980$ es la estabilización đel número absoluto de nifíos menores de 15 años y la reducción de su proporción sobre el total de la población desde un 39,6 a $32,5 \%$. Mientras en 1970. el censo corregido de ese año dio 3.567 .980 menores de 15 años, nuestra estimación para 1980 , contabilizando los nacidos vivos a partir de 1966 y 
los fallecidos en cada año hasta 1980, alcanza a 3.675 .472 , ligeramente mayor que la estimación del Instituto Nacional de Estadistica de 3.613.000 menores de 15 años. Mientras la población adulta aumentó en un $29 \%$ entre 1970 y 1980 , la población infantil lo hizo sólo en un 1\% (Tabla 1). E] fenómeno deriva del significativo descenso de los nacimientos en los últimos años; mientras en 1963 hubo 310.000 nacimientos vivos $37,7 \%$ por $\mathrm{mil}$ habitantes) el número bajó a 253.000 en 1980 $(22,8$ por mil habitantes).

Tabla 1 ,

Población Chilena según edad, 1970 y 1980.

\begin{tabular}{|c|c|c|c|c|}
\hline $\begin{array}{l}\text { Edades } \\
\text { (años) }\end{array}$ & $\begin{array}{l}\text { Población } \\
1970\end{array}$ & $\begin{array}{l}\text { (miles) } \\
1980\end{array}$ & Variación & n $\%$ \\
\hline $\begin{array}{c}\text { Menos de } 1 \\
1 \text { a } 4 \\
5 \text { a } 9 \\
10 \text { a } 14\end{array}$ & $\begin{array}{r}261 \\
944 \\
1.236 \\
1.127\end{array}$ & $\begin{array}{r}253 \\
1.012 \\
1.164 \\
1.184\end{array}$ & $\begin{array}{l}\text { Menos } \\
\text { Más } \\
\text { Menos } \\
\text { Menos }\end{array}$ & $\begin{array}{l}3,1 \% \\
7,2 \% \\
5,8 \% \\
5,1 \%\end{array}$ \\
\hline $\begin{array}{l}\text { Menores de } 15 \\
15 \text { affos y más }\end{array}$ & $\begin{array}{l}3.568 \\
5.801\end{array}$ & $\begin{array}{l}3.613 \\
7.491 \\
\end{array}$ & $\begin{array}{l}\text { Más } \\
\text { Más }\end{array}$ & $\begin{array}{r}1,3 \% \\
29,1 \% \\
\end{array}$ \\
\hline TOTAL & 9.369 & 11.104 & Más & $18,5 \%$ \\
\hline
\end{tabular}

\section{2) El riesgo de muerte infantil}

\section{a) Riesgos según edad}

En los últimos 60 años el riesgo de muerte de los niños ha decrecido en forma sistemática en Chile $y$ en el caso de la mortalidad infantil (menores de un af̃o) desde valores de 248 por 1.000 nacidos vivos en 1920 a 27,2 en 1981. En la Tabla 2 se presenta la evolución del riesgo en diversas edades entre 1960 y 1980 . Como puede apreciarse las tasas de mortalidad de los niños han caído en una mayor proporción que las de la población general. Particularmente llamativo es el descenso absoluto y proporcional observado entre el primer mes y los 4 años de vida; la reducción es menor, proporcionalmente, para la mortalidad neonatal y la del niño escolar, to que tiene proba. blemerite diferente explicación. La mortakidad escolar presentaba tasas bajas, que en general son más dificiles de reducir, y la principal causa de muerte, los accidentes, no han disminuido en forma significativa; en cambio, la menor reducción en la mortalidad neonatal obedece a causas mucho más complejas, en que juegan el tipo y calidad de recursos humanos $y$ equipamientos $y$, paradojalmente, la mayor proporción de recién naciłos de alto riesgo por bajo peso extremo que nacen vivos y que mueren precozmente.

La característica más destacada de la evolución de la mortalidad infantil chilena, en comparación con el resto de Latinoamérica, es la significativa mayor velocidad de descenso. Si se considera, por ejemplo, el período 1960--1977, el descenso chile-
Tabla 2.

Riesgos de Muerte según grupos de edad (Por 1.000 habitantes de igual edad) Chile, 1960 a 1980

\begin{tabular}{|c|c|c|c|c|c|c|}
\hline & 1960 & 1965 & 1930 & 1975 & 1980 & $\begin{array}{l}\text { Varición } \\
19701980 \\
{ }_{\alpha} 1980\end{array}$ \\
\hline Mor:alidad General (1) & 22.3 & 10,8 & 8,9 & 7.2 & 6,6 & Меле $26 \%$ \\
\hline Mortunataldad (2) & 24,6 & 22.6 & 20,3 & 16,7 & 9.6 & Menas 53 \% \\
\hline Mor'stidad intżntil (2) & 120.3 & 95.4 & 79,3 & 55.4 & 今ิ1.8 & Menos $50 x$ \\
\hline $\begin{array}{l}\text { Merortes de 2B diss } \\
\text { (neonslat) (2) }\end{array}$ & 35.2 & 33.5 & 31.3 & 24.8 & 16,3 & Menos $48 *$ \\
\hline Uno a II mescs 12 i & 85.1 & 61.9 & 48,0 & 30.6 & I5.5 & Senor $68 \mathrm{x}$ \\
\hline Uno a 4 añ (3) (3) & 9.6 & 5.6 & 3,9 & 2.2 & $t, 3$ & Menos $67 / 4$ \\
\hline$S_{2} \mid 4$ *hos $(3)$ & 1,4 & 1,0 & 0.9 & 0.7 & 0.6 & Menes $33 *$ \\
\hline \multicolumn{7}{|c|}{ 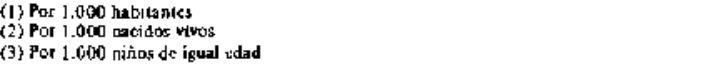 } \\
\hline
\end{tabular}

no de la mortalidad infantil fue de un $60 \%$ con una reducción de 73 puntos (tasas de 120 y 47 para 1960 y 1977) mientras Latinoamérica disminuyó sólo en un 30\% correspondiente a un descenso de 21 puntos (tasas de 69 y 48 por mil para 1960 y 1977) (57).

Los valores actuales de mortalidad infantil en Chile (27 por mil en 1981) sitúan al país muy por debajo del promedio mundial de las naciones con información completa y confiable que es de 63 por mil, aunque todavía lejana de los valores medios de Europa ( 18 por mil) o América del Norte (15 por mil).

La Tabla 3 muestra la evolución de la mortalidad infantil, separada en mortalidad de menores de 28 días (mortalidad neonatal) y de uno a 11 meses (mortalidad infantil tardía). La disminución del riesgo observada en la década del setenta y particularmente en el periodo 1975-1980 es superior a la del decenio anterior, pese a que la experiencia internacional revela que a menores valores más difícil resultan reducciones subsiguientes. Como resultado del mayor descenso de la mortalidad infantil tardia que de la mortalidad neonatal, esta última representa proporciones sistemáticamente mayores del riesgo infantil del primer afio de vida, lo que se observa en todos los países que reducen sus mortalidad infantil.

El riesgo de muerte durante el primer ano de vida ha variado en forma diversa según el momento que se considere. En la Tabla 4 puede apreciarse que las menores reducciones proporcionales del riesgo aparecen para las muertes ocurridas en el primer día y en la primera semana de vida, en comparación con otros momentos del primer mes de la existencia. Durante los 11 meses restantes del primer año se aprecia que las mayores ganancias son paralelas al número de meses de vida del lactante.

b) Mortalidad infantil por regiones del país

La disminución del riesgo de muerte infantil se aprecia en todas las regiones del país. En la 
Tabla 3.

Mortalidad Infantil en Chile, 1960 a 1980

\begin{tabular}{ccccc}
\hline Año & $\begin{array}{c}\text { Mortalidad } \\
\text { infantil } \\
\text { (MD }\end{array}$ & $\begin{array}{c}\text { Tasas por 1.000 nacidos vivos } \\
\text { Mortalidad } \\
\text { neonatal } \\
\text { (MNN) }\end{array}$ & $\begin{array}{c}\text { Mortalidad } \\
\text { tardía } \\
(\mathbf{M T})\end{array}$ & $\begin{array}{c}\text { Razón } \\
\text { MNN/MI }\end{array}$ \\
\hline 1960 & 120,3 & 35,2 & 85,1 & .29 \\
1965 & 95,4 & 33,5 & 61,9 & .35 \\
1970 & 79,3 & 31,3 & 48,0 & .40 \\
1975 & 55,4 & 24,8 & 30,6 & .45 \\
1980 & 31,8 & 16,3 & 15,5 & .51 \\
\hline
\end{tabular}

Disminución en los periodos

\begin{tabular}{|c|c|c|c|c|c|c|}
\hline \multirow[t]{2}{*}{ Períodos } & \multicolumn{2}{|c|}{ Mortalidad infantil } & \multicolumn{2}{|c|}{ Mortalidad neonatal } & \multicolumn{2}{|c|}{ Mortalidad tardia } \\
\hline & Puntos & $\%$ & Puntos & $\%$ & Puntos & $\%$ \\
\hline $1960-65$ & 24,9 & 21 & 1,7 & 5 & 23,2 & 27 \\
\hline $1965-70$ & 16,1 & 17 & 2,2 & 7 & 13,9 & 23 \\
\hline $1970-75$ & 23,9 & 30 & 6,5 & 21 & 17,4 & 36 \\
\hline $1975-80$ & 28,2 & 51 & 8,5 & 34 & 15,1 & 49 \\
\hline
\end{tabular}

Tabla 4.

Mor talidad Infan til según momento de vida en el Primer Año Chile. 1960-1980 (por 1.000 nacidos vivos)

\begin{tabular}{lrrrrrr}
\hline $\begin{array}{l}\text { Perícto } \\
\text { de ta vida }\end{array}$ & 1960 & 1965 & 1970 & 1975 & 1980 & $\begin{array}{c}\text { AN O S } \\
\text { \%de } \\
\text { variación } \\
1970-1980\end{array}$ \\
\hline 1er. dia & 8,6 & 9,8 & 9,0 & 7,9 & 6,9 & $-23 \%$ \\
1a. semana & 20,3 & 21,3 & 19,9 & 16,9 & 12,7 & $-36 \%$ \\
2a. semana & 5,5 & 4,8 & 4,4 & 3,1 & 1,6 & $-64 \%$ \\
3a. semana & 5,1 & 4,3 & 3,8 & 2,5 & 1,2 & $-68 \%$ \\
4a. semana & 4,4 & 3,0 & 3,3 & 2,4 & 0,9 & $-74 \%$ \\
\hline 20 y Jer. mes & 27,9 & 23,2 & 17,8 & 12,6 & 7,2 & $-60 \%$ \\
$4^{\circ}$ a $60^{\circ}$ mes & 27,9 & 22,2 & 17,5 & 11,1 & 5,2 & $-70 \%$ \\
70 a 90 mes & 16,6 & 10,4 & 8,2 & 4,5 & 2,1 & $-74 \%$ \\
$10^{\circ}$ a I20 mes & 10,6 & 6,0 & 4,5 & 2,5 & 1,0 & $-78 \%$ \\
\hline
\end{tabular}

Tabla 5, tas regiones chilenas han sido ordenadas en forma decreciente de acuerdo a la proporción de población urbana que vive en las regiones ya que, históricamente, las zonas rurales presentan significativos mayores riesgos de muerte. Si se compara en la Tabla 5 la situación de las regiones con mayor proporción de población urbana (I, II, III, V, XII y Metropolitana) y las de menor proporción (VI, VII, IX y X Regiones) se advierte que, en términos generales, en 1970 la mortalidad infantil de las primeras era el doble de las últimas con unos 50 puntos de diferencia. En 1980 las diferencias se han reducido notablemente (alrededor de 12 puntos de diferencia) registrándose escasas diferencias especialmente en la mortalidad neonatal. En otros términos, si bien el riesgo rural continúa siendo superior, las diferencias se han atenuado ostensiblemente tanto en términos absolutos como proporcionales, lo que podría estar relacionado con mejorías del número de personal, proporcionalmente mayores en las regiones más rurales (2).

\section{3) La demanda de bospitalización y de consulta}

La última información disponjble relativa a hospitalizaciones infantiles es la de 1975. Por este motivo se ha comparado la evolución entre 1965 y 1975 (Tabla 6). Puede apreciarse que en el curso de ese decenio las hospitalizaciones infantiles aumentaron de 152.349 a 233.563 , número equivalente a 47,2 y 62,3 hospitalizaciones por cada 1.000 niños. Este aumento global de las hospitalizaciones, de $32,0 \%$, fue significativamente superior en el caso de las hospitalizaciones de recién nacidos y lactantes menores que aumentaron en $219 \%$ y $64 \%$, respectivamente. Se observa igualmente que la letalidad de los hospitalizados dísminuyó a la mitad. La situación infantil contrasta con la observada en los mayores de 15 años cuya tasa de hospitalización disminuyó durante el período - por decrecimiento del número absoluto de partos- y cuya letalidad se mantuvo estable sin apreciarse mejorías.

La frecuencia de consulta infantil en el Sistema Nacional de Servicios de Salud, que absorbe la mayoría de las producidas en el pais, ha tenido un incremento similar al observado para las hospitali- 
Tabla 5.

Variación de la Mortalidad Infantil en las regiones chilenas. 1970-1980

\begin{tabular}{|c|c|c|c|c|c|c|c|c|c|c|c|c|c|}
\hline \multirow[b]{2}{*}{ Regiones } & \multirow{2}{*}{$\begin{array}{c}\text { \%ode po- } \\
\text { blación } \\
\text { urbana } \\
1980\end{array}$} & \multicolumn{3}{|c|}{ An̂o 1970} & \multicolumn{3}{|c|}{ Año 1980} & \multicolumn{6}{|c|}{ Variación del período $1970-1980$} \\
\hline & & M.1. & $M N N$ & M.T. & M.l. & $\mathbf{M N N}$ & M.T. & M.i. & $\begin{array}{l}\text { Puntos } \\
\text { MNN }\end{array}$ & M.T. & M.. . & $\begin{array}{c}\text { Porcentual } \\
\text { MNN }\end{array}$ & M.T. \\
\hline CHILE & 80 & 79 & 31 & 48 & 32 & 16 & 16 & 47 & 15 & 32 & 60 & 48 & 69 \\
\hline II Antofagasta & 97 & 71 & 30 & 41 & 35 & 20 & 15 & 36 & 10 & 26 & $S 1$ & 33 & 63 \\
\hline Metropolitana & 96 & $\$ 3$ & .22 & 31 & 22 & 11 & 11 & 31 & 11 & 20 & 58 & 50 & 65 \\
\hline I Tarapaćá & 93 & 55 & 26 & 29 & 27 & 17 & 10 & 28 & 9 & 19 & 51 & 35 & 66 \\
\hline Il Atacama & 90 & 93 & 36 & 57 & 31 & 18 & 13 & 62 & 18 & 44 & 66 & 50 & 77 \\
\hline V Valparaiso & 89 & 58 & 25 & 33 & 31 & 17 & 14 & 27 & 8 & 19 & 46 & 32 & 58 \\
\hline XII Mapallanes & 88 & 37 & 22 & 15 & 22 & 11 & 11 & 15 & 11 & 4 & 41 & 50 & 27 \\
\hline VIII Bio-Bío & 75 & 103 & 37 & 66 & 44 & 22 & 22 & 59 & 15 & 44 & 57 & 41 & 67 \\
\hline XI Aysén & 70 & 76 & 29 & 47 & 39 & 19 & 20 & 37 & 10 & 27 & 49 & 34 & 57 \\
\hline W Coquimbo & 68 & 90 & 35 & 45 & 50 & 24 & 26 & 40 & 11 & 19 & 44 & 31 & 42 \\
\hline 1X Araucanía & 59 & 316 & 42 & 74 & 54 & 26 & 28 & 62 & 16 & 46 & $\$ 3$ & 38 & 62 \\
\hline$X$ Los Lagos & 59 & 126 & 51 & 75 & 42 & 20 & 22 & 84 & 31 & 53 & 67 & 60 & 71 \\
\hline VlI Maule & 55 & 100 & 37 & 63 & 37 & 19 & 18 & 63 & 18 & 45 & 63 & 49 & 71 \\
\hline VI O'Higgins & 53 & 85 & 33 & 52 & 35 & 20 & 15 & 50 & 13 & 37 & 50 & 39 & 71 \\
\hline
\end{tabular}

Tabla 6.

Frecuencia de Hospitalización de la Población Infantil.

1965 y 1975

\begin{tabular}{|c|c|c|c|c|c|c|c|}
\hline \multirow[b]{2}{*}{ Edades } & \multicolumn{2}{|c|}{$\begin{array}{c}\text { Número de } \\
\text { hospitalizaciones }\end{array}$} & \multicolumn{2}{|c|}{ Tasa por 1.000} & \multirow{2}{*}{$\begin{array}{l}\text { Variación } \\
\text { de las } \\
\text { tasas } \\
(\%)\end{array}$} & \multicolumn{2}{|c|}{ Letalidad (\%) } \\
\hline & 1965 & 1975 & 1965 & 1975 & & 1965 & 1975 \\
\hline Menos de 28 días & 18.365 & 48.808 & 59,6 & 190.2 & Más $21 \%$ & 13,4 & 8,1 \\
\hline 28 dias a 11 meses & 41.211 & 56.330 & 133,8 & 219,5 & Más $64 \%$ & 13,8 & 5,1 \\
\hline Uno a 4 años & 36.895 & 56.028 & 47,1 & 54,8 & Más 16\% & 4,6 & 1,8 \\
\hline 5 a 9 años & 28.371 & 36.815 & 25,4 & 29.7 & Más $17 \%$ & 1,7 & 1,0 \\
\hline 10 a 14 años & 27.507 & 35.582 & 27,0 & 29,0 & Más $7 \%$ & 1,4 & 0,9 \\
\hline Menotes de 15 años & 152.349 & 233.563 & 47,2 & 62,3 & Más $32 \%$ & 7,0 & 3,6 \\
\hline 15 años y más & 661.687 & 720.935 & 125.9 & 111,8 & $-11,2 \%$ & 2,5 & 2,5 \\
\hline TOTAL & 814.036 & 954.498 & 95,9 & 93,6 & $-2,4 \%$ & 3,3 & 2,8 \\
\hline
\end{tabular}

zaciones infantiles (Tabla 7). Mientras en 1970 se dieron 3,5 millones, en 1980 el número subió a 6,3 millones duplicándose casi las tasas por habitante. El aumento en la frecuencia de consultas es proporcionalmente menor en la Región Metropolitana que en las regiones con alta proporción de población urbana (I, II, V y XII Regiones) y en éstas que en aquelias regiones con la mayor proporción de población rural.
4) Notificación de enfermedades infecciosas trasmisibles

En la información existente respecto a notificación no es posible saber cuántos casos se produjeron en menores de 15 años; sin embargo, como las patologías consideradas afectan mayoritariamente a la población infantil los datos globales pueden servir de indicación a lo ocurrido en los 
Tabla 7.

Frecuencia de Consulta Infantil. Sistema Nacional de Servicio de Salud.

1970 y 1980

\begin{tabular}{|c|c|c|c|c|c|}
\hline \multirow{2}{*}{$\begin{array}{l}\text { Tipo de } \\
\text { consulta }\end{array}$} & \multicolumn{2}{|c|}{$\begin{array}{l}\text { Número de consultas } \\
\text { (miles) }\end{array}$} & \multicolumn{2}{|c|}{ Consultas por habitantes } & \multirow{2}{*}{$\begin{array}{c}\text { Variación de tasas } \\
\text { \% }\end{array}$} \\
\hline & 1970 & 1980 & 1970 & 1980 & \\
\hline Infantiles & 3.550 & 5.281 & 0,99 & 1,74 & Mús $76 \%$ \\
\hline Maternales & 487 & 1.101 & $1,87^{*}$ & $4,33 *$ & Más $132 \%$ \\
\hline Adultos & 4.877 & 8.595 & 0,84 & 1,15 & Más $37 \%$ \\
\hline TOTAL & 10.782 & 15.977 & 1,15 & 1,44 & Más $25 \%$ \\
\hline \multicolumn{6}{|c|}{ Consultas infantiles } \\
\hline \multicolumn{6}{|l|}{ Región } \\
\hline Metropolitana & 2.037 & 2.535 & 1,33 & 1,74 & Más $31 \%$ \\
\hline \multicolumn{6}{|l|}{ I, II, V y XII } \\
\hline Regiones & 728 & 1.146 & 1,37 & 1,91 & Más $39 \%$ \\
\hline Otras Regiones & 1.527 & 2.600 & 1,01 & 1,67 & Más 65\% \\
\hline
\end{tabular}

* Por nacido vivo.

Tabla 8.

Notificación de Enfermedades infecciosas trasntisibles en Chile 1970-1980

\begin{tabular}{|c|c|c|c|c|c|c|}
\hline \multirow{2}{*}{$\begin{array}{l}\text { Tipo de } \\
\text { enfermedades }\end{array}$} & \multicolumn{3}{|c|}{$\begin{array}{l}\text { Número de notificaciones } \\
\text { (miles) }\end{array}$} & \multicolumn{3}{|c|}{ Tasa por 100.000 habitantes } \\
\hline & 1970 & 1975 & 1980 & 1970 & 1975 & 1980 \\
\hline TOTAL & 69,3 & 69,9 & 83,2 & 740 & 681 & 750 \\
\hline $\begin{array}{l}\text { Gripe } \\
\text { Total excluyendo } \\
\text { gripe }\end{array}$ & $\begin{array}{l}17,2 \\
52,1\end{array}$ & $\begin{array}{r}5,5 \\
64,4\end{array}$ & $\begin{array}{l}14,9 \\
68,3\end{array}$ & $\begin{array}{l}183 \\
557\end{array}$ & $\begin{array}{r}53 \\
628\end{array}$ & $\begin{array}{l}230 \\
519\end{array}$ \\
\hline $\begin{array}{l}\text { Respiratorias (1) } \\
\text { Digestivas (2) }\end{array}$ & $\begin{array}{r}17,1 \\
6,9\end{array}$ & $\begin{array}{l}27,8 \\
10,8\end{array}$ & $\begin{array}{l}26,1 \\
15,3\end{array}$ & $\begin{array}{r}182 \\
74\end{array}$ & $\begin{array}{l}276 \\
106\end{array}$ & $\begin{array}{l}235 \\
138\end{array}$ \\
\hline Venéreas (3) & 1,4 & 5,3 & 8,2 & 16 & 51 & 74 \\
\hline Zoonosis (4) & 1,1 & 1,0 & 0.7 & 11 & 9 & 7 \\
\hline Otras (5) & 0,5 & 0,4 & 0,5 & 5 & 4 & 4 \\
\hline $\begin{array}{l}\text { Controlables pò } \\
\text { vacunación (6) }\end{array}$ & 25,1 & 11,4 & 6,9 & 267 & 111 & 62 \\
\hline
\end{tabular}

(1) Escarlatina, fiebre reúmatica, meningitis meningocócica, parotiditis epidémica, rubeola, varicela.

(2) Amebiasis, disenterias, hepatitis, tifoidea y paratifoidea

(3) Sífilis

(4) Hidatidosis, brucelosis, carbunco, triquinosis, rabia humana

(5) Encefalitis, erisipela, té tanos, tifo, tracoma

(6) Difteria, poliomielitis, sarampión y tos ferina 
nirlos. La tasa total de notificación ha variado escasamente aún cuando aparecen tendencias diversas para las enfermedades de tipo digestivo $y$ venéreo, que aumentan durante la década del setenta, y las zoonosis a los problernas susceptibles de control por vacunaciones, que disminuyen (Tabla 8).

5) Morbilidad infantil percibida por las madres $y$ responsables de los niños

Una importante proporción de la patología in. fantil no recurre a las distintas fuentes de atención médica (42). Por esta razón, la cuantificaciôn de la patología a través del número de consultas no expresa realmente la situación existente. Esta in. formación sólo puede ser obtenida a través de encuestas poblacionales (18) (39) (42) (43) (44). Dados los diversos modelos y definiciones usados por los investigadores suele ser difícil la comparación de los resultados. Ello sólo es posible en el caso del Gran Santiago de acuerdo a los resultados de Hall (21) en 1968 y los de Medina y Kaempffer en 1978 (42) (43) (44), por cuanto estè último estudio utilizó intencionadamente similares criterios y definiciones. Por ello es posible señalar que en Santiago el número anual de episodjos de enfermedades percibidas por las personas se redujo de 4,5 a $4,1(-8,2 \%)$ y $q u e$ en el caso de los menores de 15 aftos el fenómeno habría sido una disminución de 4,1 a 2,9 episodios anuales por niño $(-30 \%)$ de acuerdo a las estimaciones basadas en extrapolaciones de los datos globales.

\section{6) Los grandes problemas de salud infantil}

Los últimos datos disponibles revelan que, por una parte, se ha producido un significativo aumento de las consultas (Tabla 1) y de las atenciones hospitalarias, paralelamente a una impresionante disminución del riesgo de muerte infantil durante la década de 1970 (Tabla 9). En el caso de las

Tabla 9.

Modificaciones de los principales riesgos de hospitalización y muerte de los niños menores de 15 años en chile

Tasas por 100.000 habitantes

\begin{tabular}{|c|c|c|c|c|}
\hline \multirow[t]{2}{*}{ Problemas } & \multicolumn{2}{|c|}{ Tasas de hospitalización } & \multicolumn{2}{|c|}{ Tasas de mortalidad } \\
\hline & 1965 & 1975 & 1970 & 1980 \\
\hline TOTAL & 4.717 & 6.381 & 741 & 290 \\
\hline Infecciosos trasmisibles & 1.880 & 1.195 & 180 & 30 \\
\hline Perinatales & 542 & 716 & 123 & 87 \\
\hline Respiratorios & 1.155 & 1.597 & 254 & 44 \\
\hline Accidentes y violencias & 432 & 569 & 45 & 40 \\
\hline Otros problemas & 540 & 2.033 & 112 & 60 \\
\hline Congénitas & 168 & 271 & 27 & 29 \\
\hline
\end{tabular}

Tabla 10.

Importancia relativa de diversos problemas como primeras cinco causas de muerte en la infancia.

Chile. 1970 y 1980 . Porcentajes sobre el total de muertes de cada grupo de edad.

\begin{tabular}{|c|c|c|c|c|c|c|c|c|c|c|}
\hline \multirow[t]{2}{*}{ Problemas } & \multicolumn{5}{|c|}{ AÑ 1970} & \multicolumn{5}{|c|}{ AÑO 1980} \\
\hline & Total & -1 año & $1-4$ & $5-9$ & $10-14$ & Toral & -1 año & $1-4$ & $5-9$ & $10-14$ \\
\hline Total de muertes & 26446 & 20750 & 3684 & 1160 & 852 & 10648 & 8072 & 1267 & 683 & 626 \\
\hline Respiratorias & $34 \%$ & $35 \%$ & $37 \%$ & $21 \%$ & $14 \%$ & $15 \%$ & $16 \%$ & $17 \%$ & 9 & 9 \\
\hline Infecciosas & 24 & 25 & 23 & 17 & 12 & 10 & 11 & 11 & 6 & 8 \\
\hline Perinatales & 17 & 21 & & & & 30 & 39 & & & \\
\hline Accidentes & 6 & 1 & 17 & 31 & 38 & 14 & 6 & 33 & 43 & 43 \\
\hline Congénitas & 4 & 4 & & & & 10 & 12 & & & \\
\hline Nerviosas & & & 5 & 6 & 5 & & & 7 & 8 & 7 \\
\hline Nutricionales & & & 4 & & & & & & & \\
\hline Tumores & & & & 8 & 8 & & & 6 & 12 & 12 \\
\hline Otras causas & 15 & 14 & 14 & 17 & 23 & 21 & 16 & 26 & 22 & 21 \\
\hline
\end{tabular}


enfermedades infecciosas, la declinación simultánea de los egresos hospitalarios y de las muertes sugiere una menor producción de casos de estas patologías. El aumento de los egresos y disminución de las muertes se observa para los problemas perinatales, respiratorios y traumáticos. La patologia de origen congénito, en cambio, muestra aumento de egresos y de muertes sugiriendo un aumento real no controlado.

Por la diversa magnitud y velocidad con que han evolucionado los diferentes problemas de salud, se ha modificado completamente la importancia relativa de ellos. Mientras en 1970 la patologia respiratoria e infecciosa determinaba un $58 \%$ de las defunciones infantiles, en 1980 la proporción ha bajado al $25 \%$. En cambio, los problemas
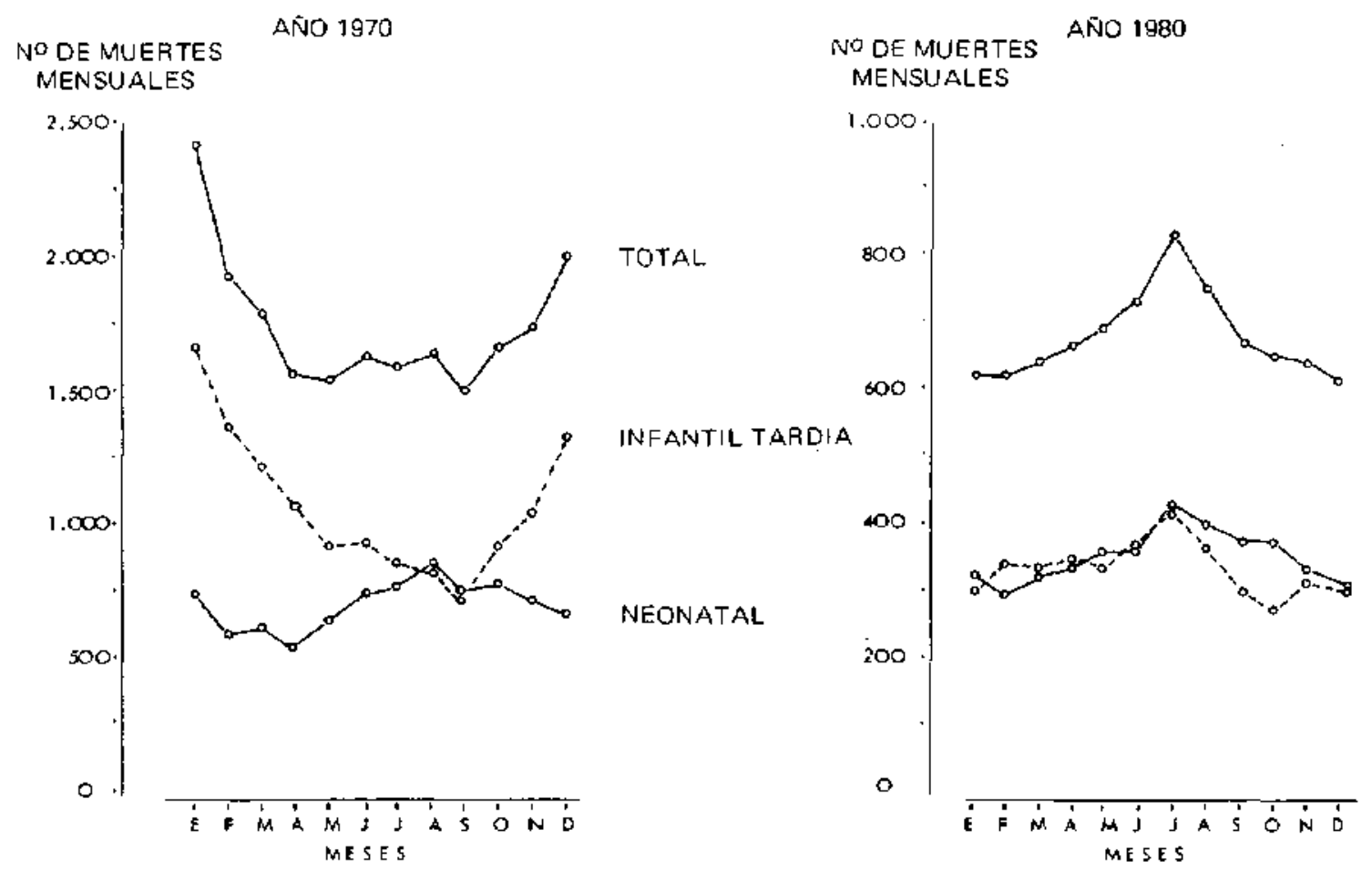

Figura 1. perinatales han aumentado su importancia relativa de 17 a $30 \%$ y los problemas congénitos de 4 a 10\%. Es también llamativo el incremento proporcional de las muertes accidentales de 6 a $14 \%$. En el cambio de situación llama la atención que, ya a partir del año de edad, la principal causa de muerte en la infancia en 1980 sean accidentes y violencias y que los tumores malignos determinen más muertes en escolares que las enfermedades infecciosas trasmisibles (Tabla 10).

En la década de 1970-1980, la mortalidad infantil chilena ha sufrido variaciones substanciales en su magnitud y composición que han determiComo se aprecia en la Figura 1, la clásica variación estacional, con incremento invernal de la mortanado cambios en la tradicional oscilación mensual.

Mortalidad Infantil según meses, 1970 y 1980.

lidad neonatal $y$ estival de la mortalidad infantil tardía entre 1 y 11 meses, se ha modificado completamente. El hecho se entiende mejor si se cuantifican los problemas que se han modificado. Entre 1970 y 1980 la mortalidad infantil chilena bajó a un $40 \%$ del valor original (de 79,3 a 31,8 por mil nacidos vivos), esto es en 47,5 puntos. Casi la mitad de este descenso deriva de la disminución de las muertes respiratorias (descenso de 22,9 puntos) $y$ algo más de un tercio, de la caída de las enfermedades infecciosas (16,6 puntos). El descenso de ambas causas explica un $83,1 \%$ de la disminución de la mortalidad infantil del periodo y el descenso de los riesgos perinatales, un $8,9 \%$ adicional.

\section{7) Factores que explican los cambios}

La frecuencia $y$ las causas de enfermedad $y$ muerte en una comunidad son el resultado de un complejo proceso de interacción en el que inter vienen las características demográficas y genéticas de la población, el medio físico; las condiciones sociales y culturales, que englobamos en la expresión genérica de nivel de vida y cuyos principales componentes vinculados a salud son la nutrición y 
el saneamiento ambiental $y$, finalmente, la cobertura y eficiencia de los sistemas de atención de salud.

Durante la década del setenta algunos de estos factores condicionantes no se modificaron como ocurre con la estructura genética de la población chilena, ya que ésta no ha sufrido procesos inmigratorios o emigratorios significativos. En cambio, han ocurrido importantes variaciones de otros factores que consideraremos a continuación.

\section{a) La situación económica durante la década del setenta}

Diversos estudios realizados en Chile èn el pasado (3) (4) (5) han enfatizado el rol de los factores socioeconómicos como determinantes del riesgo de muerte infantil, encontrando evidencias de cómo dicho riesgo desciende al elevarse el nivel socioeconómico de las familias. El estudio de la evolución de la mortalidad infantil entre 1960-1970 hecho por Mc Cormick (6) encuentra una relación negativa con el porcentaje de población urbana y positiva con el analfabetismo. Sin embargo, ya en esa época se advierte que la atención médica del parto constituía el factor más asociado a la mortalidad infantil de la época.

Como se aprecia en la Figura 2, que muestra la

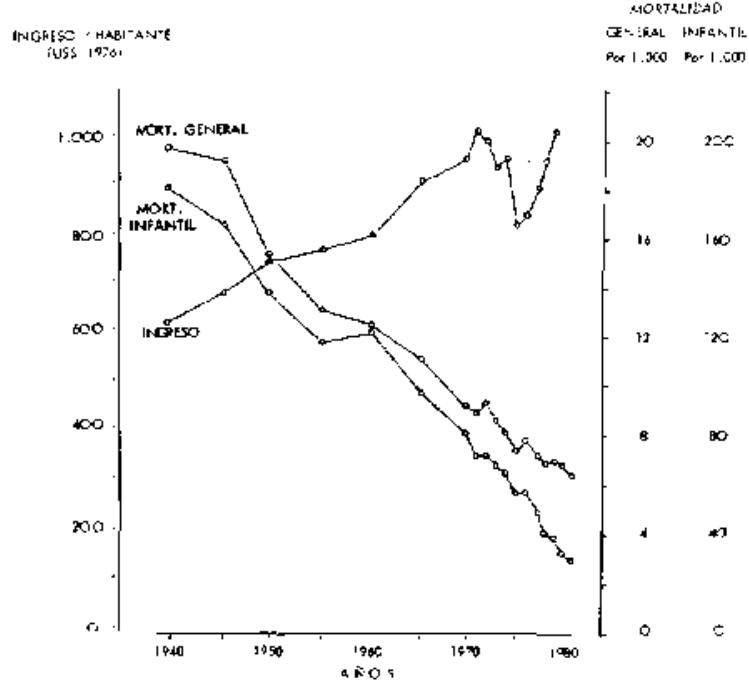

Figura 2.

Evolución del [ngreso por habitante y de la mortalidad en Chile. $1940-1980$

evolución histórica del producto nacional entre 1940 y 1980 y de la mortalidad infantil durante el mismo periodo, hasta 1970 se dio en Chile el fenómeno habitualmente observado de una relación inversa entre la situación económica y el riesgo de muerte infantil. Pero, a partir de 1970, el riesgo infantil, que muestra una tendencia declinante sostenida, se hizo enteramente independien- te de lo que sucedió con la situación económica general de estabilización global y las oscilaciones observadas durante el periodo (producto geográfjco bruto por habitante expresado en dólares de 1976; US\$ 947 en 1970 y US\$ $1.000,9$ en 1979 con ascensos y descensos agudos que llegaron hasta US\$ 818, en 1975). El estudio de las regiones muestra, igualmente, que no existe correlación entre el ingreso regional y la mortalidad infantil de las regiones del país, $(r=-0,181)$. La asociación mejora significativamente $(\mathrm{r}=-0,462)$ cuando se considera la relación entre el gasto público en salud de las regiones $y$ el riesgo infantil. De las evidencias anteriores se desprende que en el momento actual, el mejoramiento de la salud infantil en Chile no está relacionado con la mejoría de la condición económica y del nivel de vida global, sino con la actividad específica del sector salud en la atención de la población infantil.

b) La atención de salud durante la década deJ setenta

En la Tabla 11 se ha sintetizado la información disponible respecto a algunos indicadores globales del nivel de vida; actividades del sector salud y modificaciones de factores condicionantes de la situación de salud.

La tabla revela que, paralelamente a valores virtualmente estables del producto nacional por habitante durante la década de 1970-1980, la magnitud del gasto público en salud -mayoritario dentro del sector- también se mantuvo estable. Dicha estabilización de los recursos disponibles se refleja en el escaso crecimiento del total del personal del S.N.S. Dicho personal sufrió una redistribución interna con disminución de los médicos por 10.000 habitantes y aumento de enfermeras, matronas $y$ auxiliares de enfermería. En el panorama global de los médicos hacen excepción los pediatras -cuya tasa en relación a la población decrece en menor proporción-y los médicos obstetras que incrementaron significativamente. Las camas pediátricas tambiên se encuentran estabilizadas en relación con la población.

Dada la estabilidad de los recursos disponibles resulta llamativo el significativo aumento de la cobertura de consultas y egresos hospitalarios y el incremento de la dosis de vacunas colocadas y del Programa Nacional de Alimentación Complementaria, todo lo cual traduce un aumento notable de la eficiencia de las acciones de salud. Como resultado de la mayor disponibilidad de enfermeras, matronas y auxiliares de enfermeria hay un ascenso espectacular de las acciones encomendadas a este personal.

Cabe ser̂alar, finalmente, que se han producido algunos cambios que favorecen la acción en salud. Entre ellos destaca la concentración progresiva en 
ciudades de la población chilena; el mejoramiento de la condición de saneamiento y las elevadas tasas de asistencia escolar básica y media, que sirven de indicadores del nivel de instrucción al no tenerse aún los resultados detallados del censo de 1982 (Tabla 11).

\section{COMENTARIO}

El análisis de la situación de salud infantil chilena durante la década de 1970-1980 lleva a la conclusión de que el significativo mejoramiento de dicha situación aparece particularmente vinculada a la acción más extendida, el mayor número de actividades y la mayor eficiencia dernostrada por el sector salud, que permitió controlar el efecto negativo de una fluctuante condición económica. Una política de salud que definió el grupo maternoinfantil como la primera prioridad del país y que mantuvo actividades fundamentales para la salud del niño en momentos difíciles parecen ser la clave del descenso de la mortalidad infantil de 79 a 32 por 1.000 nacidos vivos: entre 1970 y 1980

\section{Tabla 11.}

Variaciones de indicadores de Nivel de Vida, actividades del Sector Salud

y otres factores vinculados a la salud

\section{SITUACION ECONOMICA}

Producto geográfico por habitante (US\$ 1976)

Tasa de desocupación en Gran Santiago (\%)

\section{ATENCION DE SALUD}

Recursos

Gasto público en salud por habitante (US\$ 1976)

Personal del S.N.S.S. (por 10.000 habitantes)

Médicos

Pediatr ia (horas x 1.000 hab.)

Obstetricia (horas por 1.000 hab.)

Enfermeras

Matronas

Nutricionistas

Auxiliares de Enfermería

Camas de pedia tría del S.N.S.S. (x 1.000 hab.)

Indicadores de cobertura

Atención profesional del parto $(\%$

Certificación médica de defunción (\%)

Actividades

Consultas médicas infantiles (por 1.000 menores de 15 años)

Egresos haspitalarios infantiles (por 1.000 menores de 15 años)

Alimentación complementaria (Kgs. por menor đe 5 años)

Dosis de vacuna (por menor de 15 años)

Usuarias de anticonceptivos en S.N.S.S. (\% de mujeres de $15-44$ años

del pais)

Consultas de enfermeras (por habjtante)

Infantiles (por menor de 15 años)

Consultas de matronas (por nacido vívo)

Consultas de auxiliar de enfermería (por hab.)

Infantiles (por menor de 15 años)

$\begin{array}{lll}1970 & 1975 & 1980\end{array}$

947

4,1

818

15,0

1009 (1)

13,1 (1)

31
51,6
4,7
0,55
0,19
1,8
1,2
0,4
17,7
0,66

22

32 (2)

53,4

3,7

0,52

0,27

2,3

1,7

0,6

20,8

0,67

81,0

91,4

81,4

90,4

$\begin{array}{cc}0,99 & 1,74 \\ 4,7(3) & 6,4(4) \\ 10,5 & 19,9 \\ 2,9 & 3,2 \\ & \\ 13,2(4) & 19,2 \\ 0,11 & 0,27 \\ & 0,71 \\ 5,2 & 13,4 \\ & 0,26 \\ & 0,53\end{array}$

FACTORES VINCULADOS A LA SALUD

Población urbana $(\%)$

Agua de cañería ( $\%$ de viviendas)

Alcantarillado (\% de viviendas)

Asistencia escolar básica ( $\%$ de niños de esa edad)

Asistencia escolar media (\% de niños de esa edad)

$\%$ de desnutrición en menores de 6 años (\%)
41

$-$
81
(1) Año 1979
(2) Ā̄o 1978
(3) Аก๊० 1965
(4) Año 1975. 
aumentó la atención profesional del parto, la cobertura de atención, la tasa de consultas y de egresos, las dosis de vacuna por nino y la magnitud de la alimentación complementaria. Paralelamente se produjo una expansión intencionada de los servicios prestados por matronas a embarazadas y recién nacidos y de enfermeras, auxiliares y nutricionistas a los ninfos de edades mayores.

La efectividad de estas acciones se vio favorecida por la notable reducción a la séptima parte en sólo 5 años, de la desnutrición infantil avanza. da (17) producto del Programa Nacional de Alimentación Complementaria, continuación con modificaciones y mejorias de una actividad iniciada en el país en los años 50, y del programa de centros cerrados de recuperación nutricional (CONIN). En el análisis del significado de los programas de nutrición cabe destacar, en primer término, la evidente rentabilidad social del PNAC (52). En la caída de la mortalidad infantil no resulta posible separar el efecto de estos programas del efecto de la atención médica propiamente tal (50). De acuerdo a estudios realizados en la Escuela de Salud Pública de la Universjodad de Chile, un $84 \%$ de la variación de la mortalidad infantil es explicada por el conjunto formado por la atención profesional del parto, horas médicas, camas pediátricas y consultas médicas a desnutridos (47).

La reducción de la mortalidad infantil en la década del setenta aparece igualmente vinculada a la significativa disminución de la natalidad chilena con reducción de las tasas de natalidad por 1.000 habitantes de 36,3 en 1965 a 27,9 en 1970 y 22,8 en 1980 . La disminución de la natalidad tiene importancia en la reducción de la mortalidad infantil por la incidencia que en ésta tiene el número de hijos o paridad, la edad a la cual la madre procrea sus hijos y el intervalo entre los partos (51). En la cuantificación de la importancia de algunos de estos factores (50) se aprecia que la disminución proporcional de las madres de 30 años y más y el aumento paralelo de las edades jóvenes explica menos del $1 \%$ del descenso de la mortalidad infantil entre 1969-1979. Un 5\% del descenso es atribuible a la significativa reducción del número de quintos hijos o más en el orden de hermandad (50). En los estudios poblacionales realizados por nosotros en el Gran Santiago (39) (42) nos había llamado la atención la menor proporción de lactantes observada en los grupos de bajo ingreso familiar $(7,1 \%)$ en comparación con los de mayor ingreso $(18,0 \%)$, hecho que traduce un mayor control de natalidad de las parejas de bajos recursos. Mjentras en 1969, un $22 \%$ de los nacidos vivos eran hijos de madres con instrucción media o superior, en 1979 la proporción había subido el $39 \%$; esta modificación en la estructura de los nacimientos explica un 26\% del descenso de la mortalidad infantil (50). En esta forma, un 32\% del descenso de la mortalidad infantil es atribuible a las modificaciones de los nacimientos, vinculados a los cambios selectivos de diferentes grupos en cuanto a planificación farniliar.

La mayor parte del cambio de la mortalidad infantil sin embargo, aparece ligada al mejoramiento específico del sector salud dada la estabilización del ingreso y del nivel de vida durante la década y el incremento de las acciones del sector. La demanda de atención médica está vinculada, đe acuerdo a nuestros estudios, a la edad de la madre y al número de nínos en la vivienda, elementos que se han modificado en la década del setenta. El número de atenciones anuales ajustado según edad de los niños en el Gran Santiago fue de 5,0 para los híjos de mujeres de 15-24 años; de 3,1 para los de madres de $25-34 ; 2,2$ para el grupo de $35-44$ affos y 1,2 para los hjjos de mujeres mayores de 45 affos. Mientras el hijo único recibia 6,3 atenciones anuales, el número se reducía a 3,1 en los grupos familiares con 2 a 4 hijos y a sólo 0,9 atenciones anuales cuando existian 5 o más niños en la vivienda (42).

Entre los cambios demográficos con eventual implicación en salud no se ha comentado suficientemente la elevada proporción de hijos lilegítimos actuales, que ha aumentado de $19,7 \%$ en 1970 a $25.7 \%$ en 1980 y que clásicamente constituyen un grupo de alto riesgo. En el momento actual, sin embargo, la mortalidad neonatal precoz es menor en los hijos de madres solteras (53), lo que equilibraría el mayor riesgo del resto del primer año de estos niños. Para la interpretación del fenómeno se desconoce la proporción de niños ilegítimos procedentes de convivencias estables con caracteristicas sociológicas de familia nuclear.

La progresiva concentración de la población chilena en ciudades ( $76 \%$ en 1970 y $81 \%$ en 1980 ) facilita igualmente la acción de salud y el hecho se refleja en la mortalidad general que fue en 1980 de 5,7 en el Gran Santiago; 6,8 para las ciudades de 100.000 habitantes a mayores y de 7,3 para el resto del territorio. Una gradiente similar se aprecia para la mortalidad infantil, la que en los estudios realizados por nosotros en 1977 (37) (38) era de 79 por mil en localidades de tamaño inferior a 1.000 habitantes; de 45 por mil en ciudades de 50 a 300.000 habitantes y de 29 por mil en el Gran Santiago.

Es posible concluir del análisis del conjunto de la información disponible que el progreso de la salud chilena durante la década del setenta se registra preferentemente en el área materna e infantil, grupo que ha sido el gran beneficiario de estos 
avances. Las mejorías en salud durante la década se vinculan mucho más con los recursos y acciones del sector salud que con la condición económica el nivel de vida general o con otros factores clásicos condicionantes de salud, lo que es coincidente con hallazgos de estudios anteriores (37) (38) que muestran que la salud infantil en Chile se correlaciona menos con el ingreso de los habitantes que con los elementos de la atención de salud, en particular con la atención profesional del parto, el nivel de cobertura y los recursos de la atención primaria.

De este modo la experiencia chilena demuestra que cuando se estabilizan o deterioran los niveles de ingresso es posible evitar la aparición de deterioros en la salud infantil cuando se mantienen los programas de atención médica, con importante énfasis en la atención primaria y la intervención nutricional. Coincidimos con otros autores (50) en que ello fue posible por la solidez de los servicios de salud chilenos (33) (57) cuya labor se ha visto facilitada por el tipo de patologia infantil presente durante la década del setenta (58) y la mayor receptividad de las madres actuales frente a los programas infantiles. El hecho deriva, a nuestro juicio, de la importante cantidad de mujeres que han alcanzado niveles de educación media, que los estudios especializados muestran como un factor de enorme significado para cambiar creencias y valores ancestrales. Por otra parte, parece evidente que los medios de comunicación de masas han tenido un papel destacado para mostrar modelos y situaciones deseables en materia de salud.

El notable descenso de la mortalidad infantil chilena, obtenido en medio de fluctuantes condi. ciones nacionales y variaciones de importancia en los planos económico, social y de organización de los servicios, no resulta paradojal si se considera que Chile vive en la actualidad en lo que podría. mos llamar la segunda fase de desarrollo en salud (Figura 3). Cuando el tiesgo de salud es muy elevado por la existencia de defectuosas condiciones ambientales, la atención médica tiene poco rendimiento para mejorar la salud por cuanto los esfuerzos se ven limitados por las graves deficiencias del nivel de vida, lo que ocurre en el momento $A$. Cuando estas limitaciones están grandemente controladas -que es lo que ocurre hoy en día en Chile-, los países se encuentran en la situación $\mathrm{B}$, momento en el cual los progresos en salud dependen fundamentalmente de mayores extensiones de la cobertura y mejoramiento de la calidad de la atención de la salud. En una etapa posterior (momento C), los progresos no dependen tanto de la atención de salud sino de significativas correcciones de tipo ecológico y modificación de hábitos y costumbres que permitan prevenir los problemas cardiovasculares, neoplásicos y traumáticos.

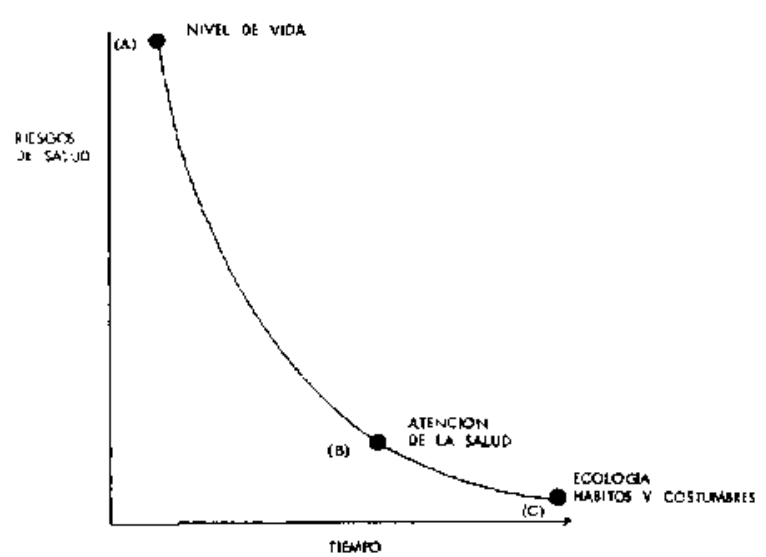

Figura 3.

Factores de importancia en el mejoramiento de da salud.

Dado el momento histórico que vive el pais y las evidencias que apoyan la mayor eficiencia de los programas de salud que la de cualquier otro factor en el descenso del riesgo infantil es que resulta indispensable la destinación de suficientes recursos económicos específicos para el sector salud y una organización y administración eficiente que concentre los esfuerzos en los problemas y en los grupos de mayor prioridad. Además de mantener y reforzar los esfuerzos actuales de atención primaria, cabe considerar que ya las muertes de recién nacidos representan la mitad de las muertes del primer año y casi un $40 \%$ de todas las muertes infantiles o que, por otra parte, accidentes y procesos reoplásicos hayan adquirido gran importancia relativa durante la vida escolar, hechos todos que obligan a emplear estrategias especiales para resolver estas nuevas situaciones que se están planteando en el conjunto de problemas de salud de los niños chilenos.

\section{RESUMEN}

Durante el decenio 1970-1980, el hecho más destacado del panorama de salud de Chile fue el gran descenso del riesgo de muerte infantil, particularmente entre el mes de edad y los 5 ar̂́os de vida, descenso que se observó a lo largo de todo el país. Paralelamente se apreció una disminución en la notificación de enfermedades trasmisibles susceptibles de control por vacunas y de la morbilidad infantil percibida por las madres en encuestas domiciliarias.

La disminución del riesgo ha determinado que la patologia respiratoria e infecciosa haya disminuido de $58 \%$ a $25 \%$ de todas las defunciones, mientras los problemas perinatales aumentaron de $17 \%$ a $30 \%$ y la patologia congénita de 4 a $10 \%$.

El descenso de la marbilidad y mortalidad en la década del setenta no se asocia a la condición económica del país sino que depende en aproxima- 
damente un $33 \%$ de cambios vinculados a la disminución de los nacimientos y en la mayor parte del $67 \%$ restante a los progresos en la atención de salud, entre los que destacan el aumento de consultas, hospitalizaciones y atención profesional del parto; la extensión de la cobertura de atención que ha superacio el $90 \%$; el incremento en las vacunaciones y la magnitud de los programas de alimentación complementaria y de centros cerrados de recuperación nutricional.

\section{REFERENCIAS}

${ }^{1}$ Medina, E. y Kaempffer, A.M.: La salud en Chile durante la década del setenta. I. Descripción de la situación. Rev. Med. Chile (por publicarse).

${ }^{2}$ Medina, E. $y$ Keempffer, A.M.: La salud en Chile durante la década del setenta. Il. Un intento de interpretación, Rev. Med. Chile (par pubticarse).

3 Behm, H.: Mortalidad infantil y nivel de vida, Edic. Universidad de Chile, 1962.

4 Boccardo, H. y Corey, G.: Medio ambiente: efectos sobte la salud. En M. Livingstone y D. Raczynski (eds) Salud Pública y Bienestar Soncial CEPLAN, Universidad Católica de Chile, 1976.

5 Legarreto, A.: Factores condicionantes de la mortalidad en la niñez. En M. Livingstone y D. Raczynski (eds). Salud Püblica y Bienestar Social, CEPLAN, Universidad Católica de Chile, 1976.

'McCormick, M., Shapiro, S. and Horn, S.D.: The relationship between infant mortality rates and medical care and socio-economic variables. Chile, 1960-1970 Internat. J. Epidemiol. 8: 145, 1979.

Instituto Nocional de Estadística.: La evolución social y económica de Chile en 7 años (1973-1980). Impr. INE, Santiago, 1980.

B Instituro Nacional de Estadistica.: Estadisticas de salud: egresos hospitalarios. 1965 a 1975.

9 Instituto Nacional de Estadistica.: Series estadísticas. Impr. INE, Santiago, 1981.

10 Instituto Nacional de Estadística: Anuarios estadísticos. 1971-1976.

11 Instituto Nacional de Estadística.: Informativos estadísticos. $1977-1981$.

12 Instifuto Nacional de Estadistica.: Compendios estadisticos. 1976-1981.

13 Ministerio de Salud. Departamento de Planificación.: Indicadores biodemográficos 1971-1981 (mịneografiado).

14 Ministerio de Salud. Departamento de Planificación.: Anuarios de Nacimientos, 1970-1980.

1 S Ministerio de Salud, Departamento de Planificacion. Anuarios de Defunciones, 1970-1980.

16 Ministerio de Salud, Departamento de Planificacion.: Anuarios de Enfermedades de Notificación Obligatoria, 1970-1980.

17 Ministerio de Salud, Departamento de Planificación. Anuarios de Atenciones y Recursos. 1970-1980.

18 Ministerio de Salud, Recursos Humanos en Chile.: un modelo de análisis. Impr. Talleres del S.N.S., 1970.

19 Ministerio de Salud.: Indicadores financieros del sector salud, 1969-1979 (mineografiado).

20 Servicio de Seguro Social:: Anuarios de Estadísticas, $1969-1979$.

21 CONPAN. Chile: estadísticas básicas en alimentación $\mathrm{y}$ nutrición. 1969-1978.

22 Colegio Médico de Chile.: Informativos sobre número y características de los médicos, 1970-1980.

23 Ochor, F.: El sector salud y sus recursos financieros: análisis de 1a década. Documento de Trabajo No 187. Corporación de Promoción Universitaria, 1979.

24 Instituto Necional de Estadistica.: Compendio estadís tico Chile 1980.

25 Escuela de Salud Arublica, Universidad de Chite.: Resúmenes de las I Jornadas Nacionales de Salud Pública. Impr. Hemusi Ltda., Santiago, 1981

26 Medina, E.: La situación de salud chilena 1970-1972. Rev. Méd. Chile 112: 150, 1974.

27 Medina, E.: Atención Médica en Chile. Rev, Salud Pública 1 : $48,1974$.

28 Medin, E.: La salud: necesidad social del mundo actual. Rev. Méd. Chile 103: 451, 1975.

29 Medinn, E.: Las condiciones de salud de los chilenos. Vida Médica 27 (3): 12, 1976.

30 Medina, E. y Cruz Coke, R.: Chilean mediche under social tevolution. New England. J. Med. 295: 193, 1976.

31 Medina, E.: El sistema de medicina curativa para empleados. Vida Médica 26 (5): 14, 1976.

32 Medina, $E_{1}$ : Los médicos chilenos $y$ las necesidades de formación. Cuad. Med. Soc. 17 (4): 7, 1976.

33 Medina, $E$.: Evolución de la salud pública en Chile en los últimos 25 años. Rev. Méd. Chìe 105: 739, 1977.

34 Medina, E.: El nivel primario de atención de salud. Rev. Méd. Chile 106: 478, 1978.

35 Medina, E.: Economía y salud. Cuad. Méd. Soc. $19(3): 5,1978$.

${ }^{36}$ Medino, E.: El desarrollo del sistema de servicios de salud de Chile. Rev. Méd. Chile 107: 1135, 1979.

37 Kaempffer, A.M. Medina, $E_{*}$, de $b$ Fuente, M. $y$ Romero, M.I.: Los problemas de salud del tecién nacido en Chile, Cuad. Méd. Soc. 17 (3): 7, 1976.

${ }^{38}$ Kaempffer, A.M. y Medino, E.: Perspectivas en la salud del niño latinoamericano. Pediatría 22: 324, 1979.

39 Kaempffer, A.M. y Medina, E.: lnvestigaciones de morbilidad infantil en el Gran Santiago. Resúmenes I Jornadas Nacionales de Salud Pública, Impr. Hemusi, Santiago, 1981.

40 Kaempffer, A.M. y Medino, A.M.: Morbilidad y atención médica del adolescente en el Gran Santiago. Resúmenes I Jornadas Nacionales de Salud Pública, Impr. Hemusi, Santiago, 1981

41 Medina, E. y Kaempffer, A.M.: Atención Médica, nivel de vida $y$ salud infantil. Resúmenes I Jornadas Nacionales de Salud Pública, Impr. Hemusi, Santiago, 1981.

42 Kaempffer, A.M. y Medin, E.: Morbilidad $y$ atención Médica infantil en el Gran Santiago. Rev. Chil. Pediatr. $51: 355,1980$.

43 Medina, E. y Kaempffer, A.M.: Morbitidad y atención médica en el Gran Santiago. Rev. Méd. Chile 107: 155, 1979.

44 Medina, E, y Kaempffer, A,M.: Características de la atención de salud en el Gran Santiago. Rev, Méd. Chile 108:937, 1980 .

ds Vargas, N., Carretero, A. y Pefie, C.: Variación de algunos factores de riesgo de la mortalidad infantil en Chile, 1971 y 1980 . Resúmenes I Jornadas Nacionales de Salud Pública, Impr. Hemusi, Santiago, 1981.

46 Solis, F., Castilko, B, y Mardones, G.: Mortalidad infantil en Chile por grupos de causas evitables en los 27 servicios de salud del país, 1979. Resúmenes I Jornadas Nacionales de Salud Pública, Impr. Hentusi Santiago, 1981.

47 Castillo, B., Mardones, G. y Solis, F.: Factores de salud asociados a la mortalidad infantil chilena por causas evitables. Rexúmenes I Jornadas Nacionales de Salud Pública, Impr. Hemusi, Santiago, 1981. 
48 Cumsille, F., Pereda, C., Legarreta, A. y cols.: Asociación entre variables socioeconómicas $y$ biodemográficas $y$ desnutrición infantil. Resúmenes J Jomadas Nacionales de Salud Pública, Impr. Hemusi, Santiago, 1981.

49 López, I., Vargas, S, y Molins, R.: Control prenatal: relación con el parto y el primer aj̃o de vida del niño. Resúmenes I Jornadas Nacionales de Salud Pública, Impr. Hemusi, Santingo, 1981.

.50 Raczynski, D. y Oyarzo, C.: ¿Por qué cae la tasa de mortalidad infantil en Chile? Colección Estudios CIEPLAN No 5, Dic. 1981.

51 Mardones, $S_{1}, F_{.}:$Análisis de 5 determinantes del nivel de salud $y$ nutrición infantil. Resúmenes I Jornadas Nacionales de Salud Pública, Impr. Hemusi, Santiago, 1981.

52 Albak, C., Infante, A., Mardones, S.F. y cols, La rentabilidad social del Programa Nacional de Alimentación Complementaria. Resúmenes I Jornadas Nacioлales de Salud Pública, Impr. Hemusi, Santiago, 1981.
53 Bania, $M$. Valderrame, $R$, y Eckhardt, E.: Características del embarazo, parto y período neonatal precoz según legitimidad. Resúmenes de 1 Jornadas Nacionales đe Salud Pública, pág. 35, Impr. Hemusi, Santiago 1981.

54 Lavados, $\boldsymbol{H}$.: (ed.) Desarrollo social y salud en Chile. Talleres Graf. Corporación, Santiago, 1979.

55 Lauados, H.: (ed.) Desartollo social y salud en Chile. 2a. parte. Bases de un sistema de salud. Talleres Graf. Corporación, Santiago, 1980.

56 Jiménez, $J_{\text {.: }}$ (ed.) Medicina social en Chile. Ediciones Aconcagua, Talleres Gráficos Corporación, Santiago, 1977.

57 Medina, $E$.: Elementos que condicionan la eficacia del sistema de Salud. Documento de Trabajo No 245. Corporación đe Promoción Universitaria, Santiago, 1981.

SB Kdempffer, A.M.: Evolución de la salud infantil entre 1952 y 1978. Rev, Mèd. Chile 10s: 719, 1977. 